\title{
On the Applicability of the Black-Scholes Model to the Inverse Quantity of Price
}

\author{
Hiroki Tahara ${ }^{1}$ \\ ${ }^{15-3-15, ~ K a m i w a k a ~ s t r e e t, ~ K o ̂ b e, ~ 651-0067, ~ J A P A N ~}$ \\ e-mail: taharahiroki2@gmail.com
}

May 28, 2020

\begin{abstract}
The aim of this article is to prove the applicability of the Black-Scholes model to the inverse quantity of price, which is the generalization of the applicability of the model to foreign currency. This issue can be formulated as the discussion whether there exists the set of real numbers as the drift and the volatility about the inverse quantity satisfying a certain system of stochastic differential equations. Solving the equations in terms of such real numbers reveals not only the existence but also that the expression is uniquely determined and has a very beautiful symmetry.
\end{abstract}

Math. Subject Classification: 39A50, 91B70, 91G30.

\section{Introduction}

According to Black and Scholes[1], the price of stocks or any financial instruments $S$ is described by a stochastic differential equation[2][3] as follows:

$$
\frac{d S}{S}=\mu d t+\sigma d W
$$



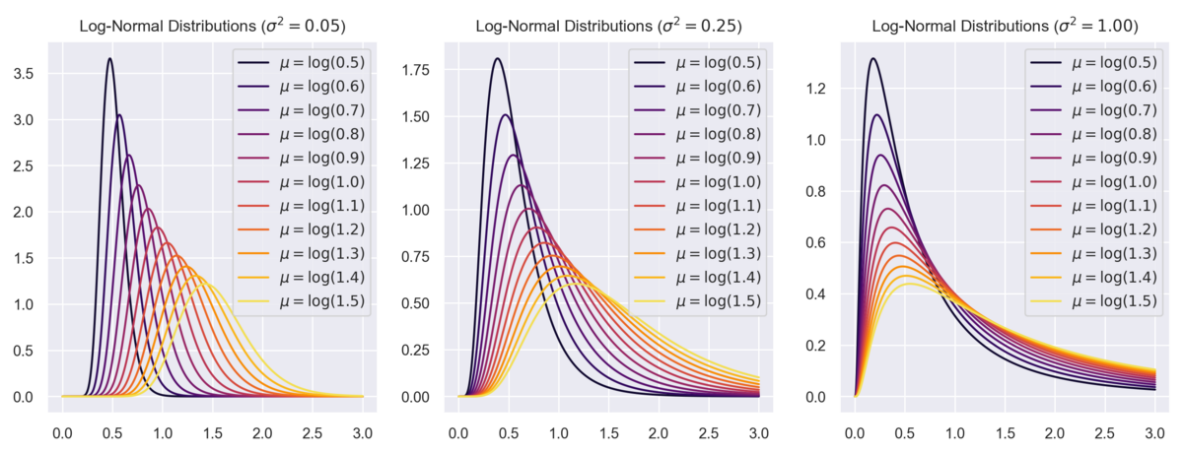

Figure 1: Instances of Log-Normal Distribution Graphs ( $\sigma^{2}$ fixed) (Koyama, 2019)[10]

referred to as the Black-Scholes model[4]. Here, $\mu$ and $\sigma$ are constants and $W$ is Wiener process[5], and the solution of this equation is as follows[6]:

$$
S=S_{0} \exp \left(\left(\mu-\frac{\sigma^{2}}{2}\right) t+\sigma W\right)
$$

derived by Itô lemma[7]. The stochastic process described by this expression is called the geometric Brownian motion[8] and it is distributed as log-normal[9] (see Figure 1.).

By the way, I have had one question: Is the Black-Scholes model applicable to foreign currency? Foreign exchange is now one of the largest financial markets in the world[11][12]. Therefore if it is not, the applicability domain of this model is not so large. Furthermore, any products can be considered as currency[13] (I think gold or silver are easy to imagine, however it is even if the product does not fulfill the three functions of currency[14]). Therefore the model seems to have to be applicable to foreign currency. However, it is nontrivial from the expressions or the graphs (Figure 1.). Therefore in order to prove it, we need a mathematical discussion which can be formulated as the issue on the applicability of the model to the inverse quantity of $S$ or the symmetry between $S$ and it, as I will explain below. 


\section{Formulation of the Issue}

The Black-Scholes model can be transformed to a form we can analyze more easy as follows:

\section{Corollary 1.}

$$
d S=\mu S d t+\sigma S d W
$$

Here, we can consider $\mu$ and $\sigma$ as the drift and volatility of the infinitesimal increase ratio of $S$ in infinitesimal time respectively[15]. After Black and Scholes, research considering them as deterministic or stochastic process have been done by Merton[16], Engle[17], etc.[18]. However here we consider them as constants. This is because the conclusion of the discussion can change depending on the definition of $\mu$ and $\sigma$ if we consider them as variables.

By the way, let $S[\mathrm{~A} / \mathrm{B}]$ be the price of foreign currency A from the view of domestic currency $B$ (i.e. per $1[B]$ ), the price of $B$ seen from $\mathrm{A}$ (i.e. per $1[\mathrm{~A}]$ ) can be expressed as follows:

\section{Definition 2.}

$$
\beta:=S^{-1}
$$

Therefore the issue on the applicability of the Black-Scholes model can be fomulated as the discussion on the symmetry between $S$ and $\beta$ as: Does $\left(\mu^{\prime}, \sigma^{\prime}\right) \in \mathbb{R}^{2}$ satisfying the system of equations below exist?

$$
\left\{\begin{array}{l}
d S=\mu S d t+\sigma S d W \\
d \beta=\mu^{\prime} \beta d t+\sigma^{\prime} \beta d W
\end{array}\right.
$$

I got this idea when I learned about information thermodynamics[19], therefore symbol $\beta$ from inverse temperature does not have profound meaning. 


\section{Proof of the Issue}

In order to solve the system in terms of $\mu^{\prime}$ and $\sigma^{\prime}$, we use Itô lemma[7]: Let $x$ be a stochastic process satisfying

$$
d x=f d t+g d W
$$

and $h$ be a process depending on $x$,

\section{Lemma 3.}

$$
d h=\left\{\frac{\partial h}{\partial x} f+\frac{1}{2} \frac{\partial^{2} h}{\partial x^{2}} g^{2}+\frac{\partial h}{\partial t}\right\} d t+\frac{\partial h}{\partial x} g d W
$$

This is just a result of Maclaurin series[20] under Itô rule[21]:

$$
(d W)^{2}=d t
$$

On the other hand, the uniqueness of coefficients[22]:

\section{Lemma 4.}

$$
A d t+B d W=C d t+D d W \Longrightarrow A=C \text { and } B=D
$$

is needed too. Due to this lemma, we can use coefficient comparison method for stochastic differential equations.

By the way, due to Definition 2. and (2) of the system,

$$
d\left(S^{-1}\right)=\mu^{\prime} S^{-1} d t+\sigma^{\prime} S^{-1} d W
$$

Therefore, let $x=\beta, f=\mu^{\prime} \beta, g=\sigma^{\prime} \beta$ and $h=S=\beta^{-1}$, by substituting these into Lemma 3.,

$$
\begin{aligned}
d S & =\left\{\frac{\partial S}{\partial \beta} \mu^{\prime} \beta+\frac{1}{2} \frac{\partial^{2} S}{\partial \beta^{2}}\left(\sigma^{\prime} \beta\right)^{2}+\frac{\partial S}{\partial t}\right\} d t+\frac{\partial S}{\partial \beta} \sigma^{\prime} \beta d W \\
& =\left\{-\frac{1}{\beta^{2}} \mu^{\prime} \beta+\frac{1}{2} \cdot 2 \cdot \frac{1}{\beta^{3}} \sigma^{\prime 2} \beta^{2}\right\} d t-\frac{1}{\beta^{2}} \sigma^{\prime} \beta d W \\
& =\frac{1}{\beta}\left(\sigma^{\prime 2}-\mu^{\prime}\right) d t+\frac{1}{\beta}\left(-\sigma^{\prime}\right) d W \\
& =S\left(\sigma^{\prime 2}-\mu^{\prime}\right) d t+S\left(-\sigma^{\prime}\right) d W
\end{aligned}
$$


By comparing coefficients of (1) and this, validated by Lemma 4 .,

$$
\left\{\begin{array}{l}
\mu=\sigma^{\prime 2}-\mu^{\prime} \\
\sigma=-\sigma^{\prime}
\end{array}\right.
$$

i.e.

$$
\left\{\begin{array}{l}
\mu^{\prime}=\sigma^{2}-\mu \\
\sigma^{\prime}=-\sigma
\end{array}\right.
$$

$\left(\mu^{\prime}, \sigma^{\prime}\right)$ satisfying this exists uniquely always. Therefore the issue is correct i.e. there exists the symmetry between $S$ and $\beta$ and the Black-Scholes model is applicable to the inverse quantity of price.

\section{Conclusion and Future Work}

As mentioned above, it is proved that the Black-Scholes model is applicable to the inverse quantity of price and there exists the symmetry between them. In addition to this, the relation between coefficients of two equations describing them

$$
\left\{\begin{array}{l}
\mu+\mu^{\prime}=\sigma^{2} \\
\sigma=-\sigma^{\prime}
\end{array}\right.
$$

is a symmetric expression in terms of $(\mu, \sigma)$ and $\left(\mu^{\prime}, \sigma^{\prime}\right)$. Intuitively speaking, this is very beautiful and seems to have very profound meanings. Therefore we ought to make a progress of the research focusing on this.

On the other hand, we only considered the situation when the drift and volatility are constants on this article as mentioned above. Therefore it seems to be interesting to consider in which cases the issue on the applicability and the symmetry holds.

\section{References}

[1] Black, F. \& Scholes, M. (1973). The pricing of options and corporate liabilities. Journal of Political Economy, 81.3, 637654 . 
[2] Herzog, F. (2010). Stochastic differential equations, Eidgenössische Technische Hochschule Zürich.

[3] Oksendal, B. (2013). Stochastic differential equations: an introduction with applications, Springer Science \& Business Media, (2013).

[4] Yamamoto, Y. (2009). Modeling of Risk Assets. Basics of Computational Finance, 3.1.2, 30-31.

[5] Lalley, S. \& Mykland, P. (2012). Wiener Process: Definition. Stochastic Processes II, G.1.1, 01.

[6] Sellamuthu, P. (2014). Black Scholes Option Pricing Model-Brownian Motion Approach. SSRN Electronic Journal, 11.6, 4587-4602.

[7] Whitt, W. (2007). Ito's Lemma. Stochastic Models in FE, 4.2, 07.

[8] Dunbar, S. R. (2016). Geometric Brownian Motion. Stochastic Processes and Advanced Mathematical Finance, 203, 03-15.

[9] Weisstein, E. W. (1999). Log Normal Distribution. Wolfram MathWorld.

[10] Koyama, Y. (2019). Visualization of log-normal distribution. Koyama Yuki no Burogu, 7.28, 075516.

[11] Gradojevic, N., Erdemlioglu, D. \& Gençay, R. (2017). Informativeness of trade size in foreign exchange markets. Economics Letters, 150, 27-33.

[12] Schrimpf, A. \& Sushko, V. (2019). Sizing up global foreign exchange markets. BIS Quarterly Review, (2019).

[13] H. Tahara. (2020). Economics Notes.

[14] Taylor, T. \& Greenlaw, S. A. (2016). Defining Money by Its Functions. Principles of Economics, 27, 01. 
[15] Elliott, R.J., Hunter, W. C. \& Jamieson, B.M. (1998). Drift and volatility estimation in discrete time. Journal of Economic Dynamics and Control, 22.2, 209-218.

[16] Merton, R. C. (1976). Option Pricing When Underlying Stock Returns Are Discontinuous. Journal of Financial Economics, $3.1 .2,125-144$.

[17] Engle, R. F. (1982). Autoregressive Conditional Heteroscedasticity with Estimates of the Variance of United Kingdom Inflation. Econometrica, 50.4, 987-1007.

[18] Dupire, B. (2007). A Brief History of Volatility. An Idiot's Guide to Option Pricing, 7, 50-57.

[19] H. Tahara. (2020). Information Thermodynamics Notes.

[20] Bourne, M. (2018). Maclaurin Series. Infinite Series Expansions, $2,01$.

[21] Belavkin, R. V. (2020). Ito differentiation rule. Pricing and Stochastic Calculus, 7, 01-05.

[22] H. Tahara. (2020). Stochastic Calculus Notes. 